\title{
A Numerical Solution of Troesch's Problem via Optimal Homotopy Asymptotic Method
}

\author{
M. Khalid \\ Department of Mathematical Sciences \\ Federal Urdu University of Arts, Sciences \& Techonology \\ University Road, Karachi-75300, Pakistan \\ Faheem Zaidi \\ Department of Mathematical Sciences \\ Federal Urdu University of Arts, Sciences \& Technology \\ University Road, Karachi-75300, Pakistan
}

\author{
Mariam Sultana \\ Department of Mathematical Sciences \\ Federal Urdu University of Arts, Sciences \& Technology \\ University Road, Karachi-75300, Pakistan \\ Aurangzaib \\ Department of Mathematical Sciences \\ Federal Urdu University of Arts, Sciences \& Technology \\ University Road, Karachi-75300, Pakistan
}

\begin{abstract}
Troesch's problem arose while the investigation of the confinement of a plasma column by applying radiation pressure was being conducted. It is an unstable problem in itself, with a two-point boundary value. The application of the Optimal Homotopy Asymptotic Method (OHAM) is used to attain an approximate solution for the nonlinear differential equation which provides and apt description of Troesch's problem. Opposing the other reported results, through the variational iteration method, Laplace Transform Decomposition Method, Homotopy Analysis Method, and the Homotopy Perturbation Method, the accuracy of the current solution is commendable for a remarkably wide range of values of Troesch's parameter. A conducted error analysis clearly admits the efficiency of OHAM.
\end{abstract}

\section{Keywords}

Troesch's Problem; Optimal Homotopy Asymptotic Method; Troesch's Parameter; Highest Degree of Accuracy.

\section{INTRODUCTION}

The Troesch's problem is innately unstable; a detailed exposition may be observed in the Weibel's book [1]. It originates from a system of non-linear ordinary differential equations being used in the study of a plasma column confinement by applying radiation pressure and via the gas porous electrodes theory $[2,3]$. The following equation governs Troesch problem.

$$
u^{\prime \prime}=\lambda \sinh (\lambda u)
$$

with boundary conditions $u(0)=0$ and $u(1)=1$. Because of its substantial value in many fields, researchers have paid it much attention by investigating it at length, e.g., a numerical solution derived using the Monte Carlo method [4], Shooting Method SM [5], the quasi-linearization technique [6,7], a method involving invariant embedding [8], inverse SM [9], Method of

\footnotetext{
${ }^{1}$ Corresponding author E-mail: khalidsiddiqui@fuuast.edu.pk
}

Adomian Decomposition [10], regular SM amalgamated with the technique of parameter mapping, overflow trap or the modified method of Newton's [11, 12], the Laplace transform with a modified decomposition technique [13], the hybrid asymptotic Finite Element Method [14], Variational Iteration Method VIM [15], Homotopy Perturbation Method [16] and Differential Transformed method [17]. Additionally, multiple recent international published articles in which The Troesch's Problem is addressed can be seen in many places [18-20].

In the following paper, a numerical treatment for the Troesch's problem is introduced that makes use of the OHAM, which is a brainchild of Marinca and Herisanu [21]. Several studies [22-29] have resulted in revelation positive facts about this method and attained solutions of important applications in the fields of engineering and science. Instead of a infinite series of infinite terms like other methods, the OHAM searches only for only a few terms, hardly two to three. The convergence ensuring method for OHAM is special, not to mention more rigorous. OHAM makes sure that a relatively rapid convergence takes place since it requires only two or three iterations to reach an accurate result. This is where the true power of the method lies. OHAM, unlike its peers, does not require a recurrence formula; it is an iterative technique that mostly converges to the exact solution after two or three iterations only. These iterations are conducted by simply identifying some coefficients. OHAM neither needs high-order approximations, nor does it have to use the rules established in the frame of homotopy analysis method; it is a method self-sustained that asks almost none of the "open questions" that other homotopy procedures ask.

In this paper, we express the concept of OHAM tenderers a reasonable and dependable solution to reach the solution of Troesch's problem. We offer numerical example with different values of $\lambda$ to show how efficient and accurate OHAM proves to be. Optimal homotopy asymptotic method points out its reliability and its potential for the solution of the aforementioned problem. This paper's organization has been listed. In the second section, for the sake of completeness, we review the basic idea behind OHAM 
Table 1. Calculated values of the constants $t_{i}$ of Troesch problem for $\lambda=0.5$ and

\begin{tabular}{cccc}
\multicolumn{4}{c}{1} \\
\hline$\lambda$ & $t_{1}$ & $t_{2}$ & $t_{3}$ \\
\hline 0.5 & -0.993234657663817000 & -0.000905419731585015 & 0.000872001403291403 \\
1 & -0.953708996835200000 & -0.009626585468833410 & 0.007761888913008840 \\
\hline
\end{tabular}

briefly. In the next section, which is Section 3, we put forward a variety of numerical results which verify that our new procedure allows for quality calculations, such that are comparable to those obtainable by high-tech techniques, where these are applicable while also enabling evaluations for Troesch parameter that lie well beyond those that can be treated with the current techniques. A conclusive summary is presented in Section 4.

\section{BASIC PRINCIPLES OF OPTIMAL HOMOTOPY ASYMPTOTIC METHOD}

Here we put forward the basic ideas of OHAM for solving the nonlinear differential equation, which consists of the form

$$
L(u(\zeta))+M(\zeta)+N(u(\zeta))=0 ; \quad P\left(u, \frac{d u}{d \zeta}\right)=0
$$

Where $L$ is a linear operator, $u(\zeta)$ is unknown function and $M(\zeta)$ is known function, $N$ is an non-linear operator and $P$ is boundary operator.

$(1-q)[L(u(\zeta, q))+M(\zeta)]=H(q)[L(u(\zeta, q))+M(\zeta)+N(u(\zeta, q))]$

and

$$
P\left(u, \frac{d u}{d \zeta}\right)=0
$$

where $q \in[0,1]$ is an embedding parameter, $H(q)$ is a non-zero auxiliary function for $q \neq 0$ and $H(0)=0, u(\zeta, q)$ is an unknown function. As $q$ increases from zero to one, the solution $u(\zeta, q)$ fluctuates from $u_{\circ}(\zeta)$ to the solution $u(\zeta)$, where $u_{\circ}(\zeta)$ is attained from Eq.(3) for $q=0$

$$
L\left(u_{\circ}(\zeta)\right)+M(\zeta)=0 ; P\left(u_{\circ}, \frac{d u_{\circ}}{d \zeta}\right)=0
$$

we choose the auxiliary function $H(q)$ in the form $H(q)=$ $\sum_{i=1} t_{i} q^{i}$, where $t_{i}$ are constants. Consider the solution of Eq.(3) in the form

$$
u\left(\zeta ; q, t_{i}\right)=u_{\circ}(\zeta)+\sum_{k \geq 1} u_{k}\left(\zeta, t_{i}\right) q^{k} ; \quad i=1,2,3, \ldots
$$

Substituting Eq.(5) in Eq.(3) and equating the coefficients of the same powers of $q$, we attain the governing equations of $u_{\circ}(\zeta)$ and $u_{k}(\zeta)$. Expanding $N\left(u\left(\zeta ; q, t_{i}\right)\right)$ in series with respect to the parameter $q$;

$$
N\left(u\left(\zeta ; q, t_{i}\right)\right)=N_{\circ}\left(u_{\circ}(\zeta)\right)+\sum_{k \geq 1} N_{m}\left(u_{\circ}, u_{1}, \ldots, u_{m}\right) q^{m}
$$

where $i=1,2,3, \ldots$ and $u\left(\zeta ; q, t_{i}\right)$ is given by Eq.(5). It should be strongly noted that $u_{k}$ for $k \geq 0$ are governed by linear Eq.(4) with the linear boundary conditions that come from the original problem, which can be solved without difficulty. The convergence of the series of Eq.(5) depends upon the auxiliary constants $t_{1}, t_{2}, t_{3}, \ldots$. If it is convergent at $q=1$, one has

$$
\left.u\left(\zeta, t_{i}\right)=u_{\circ}(\zeta)\right)+\sum_{k=1} u_{k}\left(\zeta, t_{i}\right)
$$

Generally speaking, the solution of Eq.(1) can be determined approximately in the form

$$
\left.u^{m}\left(\zeta, t_{i}\right)=u_{\circ}(\zeta)\right)+\sum_{k=1}^{m} u_{k}\left(\zeta, t_{i}\right) ; \quad i=1,2,3, . ., m
$$

Substituting Eq.(8) into Eq.(2), we get the following residual

$$
R\left(\zeta, t_{i}\right)=L\left(u^{m}\left(\zeta, t_{i}\right)\right)+M(\zeta)+N\left(u^{m}\left(\zeta, t_{i}\right)\right)
$$

Where $i=1,2,3, . ., m$. If $R\left(\zeta, t_{i}\right)=0$ then $u^{m}\left(\zeta, t_{i}\right)$ is likely to be the exact solution. Usually, such case will not arise for nonlinear problems, but we can minimize the following functional by least square or Galerkin Method and find values of $t_{1}, t_{2}, t_{3}, \ldots, t_{m}$

$$
K\left(t_{i}\right)=\int_{a}^{b} R^{2}\left(\zeta, t_{i}\right) d \zeta
$$

where $a$ and $b$ are two values, depending on the given problem. The unknown constants $t_{1}, t_{2}, t_{3}, \ldots, t_{m}$ can be optimally recognized from the conditions

$$
\frac{\partial K}{\partial t_{1}}=\frac{\partial K}{\partial t_{2}}=\ldots=\frac{\partial K}{\partial t_{m}}=0
$$

Through these known constants, the approximate solution (of order $m$ ) in Eq.(8) is well determined.

\section{APPLICATION OF OPTIMAL HOMOTOPY ASYMPTOTIC METHOD ON TROESCH'S PROBLEM}

From Eq.(1), we separate linear and non-linear part as $L[u]=u^{\prime \prime}$ and $N[u]=\lambda \sinh (\lambda u)$

Now, formulation of homotopy with the help of Eq.(3) gives

$$
(1-q) L[u]-H(\zeta, q)[L[u]-N[u]+M(\zeta)]=0
$$

We select an auxiliary function in the form

$$
H(\zeta, q)=t_{1} q+\left(t_{2}+t_{3} \zeta\right) q^{2}
$$

where $q$ is an embedding parameter and $t_{1}, t_{2}, t_{3}$ are constants which have to be calculated later. Now, zeroth, first and second 
Table 2. Comparison of $O H A M$ with exact solution and other reported approximate solutions for $\lambda=0.5$

\begin{tabular}{ccccccccc}
\hline$\zeta$ & Exact & This & ADM & VIM & MHPM & HPM & HAM & LDTM \\
{$[30]$} & {$[13]$} & {$[19]$} & 0.095945 \\
& & Work & {$[12]$} & {$[31]$} & 0.095944 \\
0.1 & 0.095944 & 0.095944 & 0.095938 & 0.100042 & 0.095940 & 0.095948 & 0.095 \\
0.2 & 0.192129 & 0.192129 & 0.192118 & 0.200334 & 0.192119 & 0.192136 & 0.192129 & 0.192129 \\
0.3 & 0.288794 & 0.288794 & 0.288780 & 0.301128 & 0.288781 & 0.288804 & 0.288795 & 0.288794 \\
0.4 & 0.386185 & 0.386185 & 0.386169 & 0.402677 & 0.386168 & 0.386197 & 0.386186 & 0.386185 \\
0.5 & 0.484547 & 0.484547 & 0.484530 & 0.505241 & 0.484527 & 0.484560 & 0.484549 & 0.484547 \\
0.6 & 0.584133 & 0.584133 & 0.584117 & 0.609082 & 0.584113 & 0.584146 & 0.584135 & 0.584133 \\
0.7 & 0.685201 & 0.685201 & 0.685187 & 0.714470 & 0.685182 & 0.685212 & 0.685203 & 0.685201 \\
0.8 & 0.788017 & 0.788017 & 0.788006 & 0.821682 & 0.788002 & 0.788025 & 0.788018 & 0.788017 \\
0.9 & 0.892854 & 0.892854 & 0.892848 & 0.931008 & 0.892846 & 0.892859 & 0.892855 & 0.892854 \\
\hline AARE & & $1.4 \times 10^{-9}$ & $1.2 \times 10^{-5}$ & $4.2 \times 10^{-2}$ & $1.4 \times 10^{-5}$ & $9.1 \times 10^{-6}$ & $1.1 \times 10^{-6}$ & $1.5 \times 10^{-8}$ \\
\hline
\end{tabular}

order problems have been attained by using last two equations and by equating the identical power of $q$,

$$
\begin{aligned}
q^{\circ}: & u_{\circ}^{\prime \prime}(\zeta)=0 \\
q^{1}: & \lambda t_{1} \sin \left(\lambda u_{\circ}(\zeta)\right)-u_{\circ}^{\prime \prime}(\zeta)-t_{1} u_{\circ}^{\prime \prime}(\zeta)+u_{1}^{\prime \prime}(\zeta)=0 \\
q^{2}: & \lambda t_{2} \sin \left(\lambda u_{\circ}(\zeta)\right)+\lambda t_{3} \zeta \sinh \left(\lambda u_{\circ}(\zeta)\right)+\lambda^{2} t_{1} \cosh \left(\lambda u_{\circ}(\zeta)\right) \times \\
& u_{1}(\zeta)-t_{2} u_{\circ}^{\prime \prime}(\zeta)-t_{3} \zeta u_{\circ}^{\prime \prime}(\zeta)-u_{1}^{\prime \prime}(\zeta)-t_{1} u_{1}^{\prime \prime}(\zeta)+u_{2}^{\prime \prime}(\zeta)
\end{aligned}
$$

From the above equations, these values of $u_{\circ}(\zeta), u_{1}(\zeta)$ and $u_{2}(\zeta)$ can be obtained as a solution of differential equations

$$
\begin{gathered}
u_{\circ}(\zeta)=\zeta \\
u_{1}(\zeta)=\frac{t_{1}}{\lambda}(\zeta \sinh \lambda-\sinh (\lambda \zeta)) \\
=\frac{1}{8 \lambda^{2}}\left[\left(8 \zeta \cosh (\lambda)\left[-2 t_{3}+\lambda t_{1}^{2} \sinh (\lambda)\right]+8 \cosh (\lambda \zeta)\right.\right. \\
{\left[2 t_{3}-\lambda t_{1}^{2} \zeta \sinh (\lambda)\right]+8\left(2 t_{3}(\zeta-1)+\lambda\left(t_{1}+t_{1}^{2}+t_{2}+\right.\right.} \\
\left.\left.t_{3}\right) \zeta \sinh (\lambda)\right)-8\left[\lambda\left(t_{1}+t_{1}^{2}+t_{2}+t_{3} \zeta\right)-2 t_{1}^{2} \sinh (\lambda)\right] \\
\sinh (\lambda \zeta)+t_{1}^{2}\left(-16 \zeta \sinh ^{2}(\lambda)-\lambda \zeta \sinh (2 \lambda)+\right.
\end{gathered}
$$$$
u_{2}(\zeta)=\frac{1}{8 \lambda^{2}}\left[\left(8 \zeta \cosh (\lambda)\left[-2 t_{3}+\lambda t_{1}^{2} \sinh (\lambda)\right]+8 \cosh (\lambda \zeta)\right.\right.
$$$$
\lambda \sinh (2 \lambda \zeta)]
$$

Thus, the approximate solution, as described in Eq.(8), is

$$
\begin{aligned}
u(\zeta)= & \frac{1}{8 \lambda^{2}}\left[\left(8 \zeta \cosh (\lambda)\left[-2 t_{3}+\lambda t_{1}^{2} \sinh (\lambda)\right]+8 \cosh (\lambda \zeta)\left[2 t_{3}-\right.\right.\right. \\
& \left.\lambda t_{1}^{2} \zeta \sinh (\lambda)\right]+8\left(2 t_{3}(\zeta-1)+\lambda^{2} \zeta+\lambda\left(2 t_{1}+t_{1}^{2}+t_{2}+\right.\right. \\
& \left.\left.t_{3}\right) \zeta \sinh (\lambda)\right)-8\left[\lambda\left(2 t_{1}+t_{1}^{2}+t_{2}+t_{3} \zeta\right)-2 t_{1}^{2} \sinh (\lambda)\right] \\
& \sinh (\lambda \zeta)+t_{1}^{2}\left(-16 \zeta \sinh ^{2}(\lambda)-\lambda \zeta \sinh (2 \lambda)+\right. \\
& \lambda \sinh (2 \lambda \zeta)]
\end{aligned}
$$

To obtain the unknown convergent constants $t_{1}, t_{2}, t_{3}$, "Minimize" command in Mathematica 9.0 has been used and obtain the following values (see Table 1).

In Tables 2 and 3, the numerical solution obtained by the Optimal homotopy Asymptotic method is compared with the exact solution and with the numerical solution obtained by different numerical schemes such as Adomain decomposition method [12], Laplace transform decomposition method [13], Modified homotopy perturbation method [18] homotopy perturbation method [19], homotopy analysis method [30], Variational iteration method [31] and for the case $\lambda=0.5$ and $\lambda=1.0$ respectively.

As opposed to other available solutions attained by using the homotopy perturbation method, Homotopy analysis method, Adomain decomposition method, Modified homotopy perturbation method, Variational Iteration method and Laplace transform decomposition method, the proposed solution exhibits the highest degree of precision in the results for a remarkable wide range of values of Troesch's parameter $\lambda$ [see Tables 2 and 3].

\section{CONCLUDING REMARKS}

In this paper, we derived an approximate solution for Troesch's problem by Optimal Homotopy Asymptotic Method. Besides that, we sketched a comparison between the proposed solution, numerical solution, and other approximations reported in the literature. The numerical results show that the proposed solution remains the most accurate one. In conclusion, the Optimal Homotopy Asymptotic Method is a powerful and efficient technique for the purpose of finding the approximate solutions for the Troesch's problem using a limited number of terms.

\section{ACKNOWLEDGMENT}

We thank the reviewers for their thorough efforts in editing our paper and highly appreciate the comments and constructive criticism that significantly contributed in improving the quality of the publication. The authors also thank Ms. Wishaal Khalid for proofreading our research paper.

\section{CONFLICT OF INTERESTS}

The authors declare that there is no conflict of interests regarding the publication of this paper.

\section{REFERENCES}

[1] Weibel, E.S. (1958) The plasma in magnetic field. Landshoff RKM (Ed.), Stanford University Press, Stanford. pp 60-67.

[2] Godaspow, D., Baker, B.S. (1973) A model for discharge of storage batteries, J. Electrochem. Soc., 120. pp 10051010. 
Table 3. Comparison of $O H A M$ with exact solution and other reported approximate solutions for $\lambda=1.0$

\begin{tabular}{ccccccccc}
\hline$\zeta$ & Exact & $\begin{array}{c}\text { This } \\
\text { Work }\end{array}$ & $\begin{array}{c}\text { ADM } \\
{[12]}\end{array}$ & $\begin{array}{c}\text { VIM } \\
{[31]}\end{array}$ & $\begin{array}{c}\text { MHPM } \\
{[18]}\end{array}$ & $\begin{array}{c}\text { HPM } \\
{[19]}\end{array}$ & $\begin{array}{c}\text { HAM } \\
{[30]}\end{array}$ & $\begin{array}{c}\text { LDTM } \\
{[13]}\end{array}$ \\
\hline 0.1 & 0.084661 & 0.084658 & 0.084249 & 0.100167 & 0.084382 & 0.084934 & 0.084673 & 0.084663 \\
0.2 & 0.170171 & 0.170168 & 0.169431 & 0.201339 & 0.169621 & 0.170698 & 0.170195 & 0.170175 \\
0.3 & 0.257394 & 0.257392 & 0.256415 & 0.304541 & 0.256593 & 0.258133 & 0.257430 & 0.257399 \\
0.4 & 0.347223 & 0.347224 & 0.346086 & 0.410841 & 0.346211 & 0.348117 & 0.347272 & 0.347230 \\
0.5 & 0.440600 & 0.440604 & 0.439402 & 0.521373 & 0.439442 & 0.441573 & 0.440661 & 0.440609 \\
0.6 & 0.538534 & 0.538539 & 0.537366 & 0.637362 & 0.537330 & 0.539498 & 0.538607 & 0.538546 \\
0.7 & 0.642129 & 0.642131 & 0.641084 & 0.760162 & 0.641010 & 0.642988 & 0.752690 & 0.642142 \\
0.8 & 0.752608 & 0.752607 & 0.751788 & 0.891287 & 0.751734 & 0.753268 & 0.752690 & 0.752623 \\
0.9 & 0.871363 & 0.871360 & 0.870909 & 1.032460 & 0.870884 & 0.871733 & 0.871425 & 0.871375 \\
\hline AARE & & $1.05 \times 10^{-5}$ & 0.002710 & 0.183610 & 0.002320 & 0.002040 & 0.019240 & $2.1 \times 10^{-5}$ \\
\hline
\end{tabular}

[3] Markin, V.S., Chernenko, A.A., Chizmadehev, Y.A., Chirkov, Y.G. (1966) Aspects of the theory of gas porous electrodes, in: V.S. Bagotskii, Y.B. Vasilev (Eds.), Fuel Cells: Their Electrochemical Kinetics, Consultants Bureau, New York, pp 21-33.

[4] Tsude, T., Ichida, K., Kiyono, T. (1967) Monte Carlo pathintergral calculations for two-point boundary-value problems, Numer. Math., 10. pp 100-116.

[5] Troesch, B.A. (1976) A simple approach to a sensitive twopoint boundary value problem, J. Comput. Phys., 21. pp 279-290.

[6] Miele, A., Aggarwal, A.K., Tietze, J.L. (1974) Solution of two-point boundary-value problems with Jacobean matrix characterized by large positive eigenvalues, J.Comput. Phys., 15. pp 117-133.

[7] Vemuri, V., Raefsky, A. (1979) On a method of solving sensitive boundary value problems, J. Franklin Inst., 307. pp 217-243.

[8] Jones, D.J. (1973) Solutions of Troesch's and other twopoint boundary-value problems by shooting techniques, J. Comput. Phys., 12. pp 429-434.

[9] Kubicek, M., Hlavacek, V. (1975) Solution of Troesch's two-point boundary value problem by shooting techniques, J. Comput. phys., 17. pp 95-101.

[10] Scott, M.R. (1975) On the conversion of boundary-value problems into stable initial-value problems via several invariant imbedding algorithms, Numerical solution of Boundary value problems for ODE. Aziz, A.K. (Edition), Academic Press, New York.

[11] Snyman, J.A. (1979) Continuous and discontinuous numerical solutions of the Troesch's problem, J.Comput. Appl. Math., 5. pp 171-175.

[12] Deeba, E., Khuri, S.A., Xie, S. (2000) An algorithm for solving boundary value problems, J. Comput. Phys., 159. pp 125-138.

[13] Khuri, S.A. (2003) A numerical algorithm for solving Troesch's problem, Int. J. Comput. Math., 80. pp 493-498.

[14] Chin, R.C.Y., Krasny, R. (1983) A hybrid asymptotic-finite element method for still two-point boundary value problems, SIAM J. Sci. Stat. Comput., 10(2). pp 229-243.

[15] Momani, S., Abuasad, S., Odibat, Z. (2006) Variational iteration method for solving nonlinear boundary value problems, Appl. Math. Comput., 183. pp 1351-1358.
[16] Feng, X., Mei, L., He, G. (2007) An efficient algorithm for solving Troesch's problem, Appl. Math. Comput., 189(1). pp 500-507.

[17] Chang, S.H., Chang, I.L. (2008) A new algorithm for calculating one-dimensional differential transform of nonlinear functions, Appl. Math. Comput., 195. pp 799-808.

[18] Khuri, S.A., Sayfy, A. (2011) Troesch's problem: a Bspline collocation approach, Math. Comput. Model., 54(910). pp 1907-1918.

[19] Mirmoradi, S.H., Hosseinpour, I., Ghanbarpour, S., Barari, A. (2009) Application of an approximate analytical method to nonlinear Troesch's problem, Appl. Math. Sci., 3(32). pp 1579-1585.

[20] Mohamad, A.J. (2010) Solving second order non-linear boundary value problems by four numerical methods, Eng. Tech. J., 28(2). pp 369-381.

[21] Marinca, V., Herisanu, N. (2008) Application of homotopy asymptotic method for solving non-linear equations arising in heat transfer. I. Comm. Heat Mass Trans., 35(6). pp $710-715$.

[22] Herisanu, N., Marinca, V. (2010) Accurate analytical solutions to oscillators with discontinuities and fractionalpower restoring force by means of the optimal homotopy asymptotic method. Comput. Math. Appl., 60(6). pp 16071615.

[23] Marinca, V., Herisanu, N. (2014) The optimal homotopy asymptotic method for solving Blasius equation, Appl. Maths. Comput., 231. pp 134-139.

[24] Iqbal, S., Idrees, M., Siddiqui, A.M., Ansari, A.R. (2010) Some solutions of the linear and nonlinear Klein-Gordon equations using the optimal homotopy asymptotic method, Appl. Math. Comput., 216(10). pp 2898-2909.

[25] Iqbal, S., Javed, A. (2011) Application of optimal homotopy asymptotic method for the analytic solution of singular Lane-Emden type equation, Appl. Math. Comput., 217(9). pp 7753-7761.

[26] Hashmi, M.S., Khan, N., Iqbal, S. (2012) Optimal homotopy asymptotic method for solving nonlinear Fredholm integral equations of second kind. Appl. Math. Comput., 218(22). pp 10982-10989.

[27] Khan, N., Mahmood, T., Hashmi, M.S. (2013) OHAM solution for thin film flow of a third order fluid through porous medium over an inclined plane. Heat Transfer Res., 44(8). pp 1-13. 
[28] Hashmi, M.S., Khan, N., Mahmood, T. (2013) Optimal Homotopy Asymptotic solution for thin film flow of a third order fluid with partial slip. World Appl. Sci. J. 21(12). pp $1782-1788$.

[29] Marinca, V., Herisanu, N. (2010) Determination of periodic solutions for the motion of a particle on a rotating parabola by means of the optimal homotopy asymptotic method. $\mathrm{J}$. Sound Vib. 329(6). pp 1450-1459.
[30] Hassan, H.N., El-Tawil, M.A. (2011) An efficient analytic approach for solving two-point nonlinear boundary value problems by homotopy analysis method, Mathematical Methods in the Applied Sciences, 34(8). pp 977-989.

[31] Momani, S., Abuasad, S., Odibat, Z. (2006) Variational iteration method for solving nonlinear boundary value problems, Appl. Math. Comput., 183. pp 1351-1358. 